\title{
A Time Domain based Playback User Interface for Voice Communication Systems in Mission Control Room Environments
}

\author{
Fabian Junge ${ }^{1}$, Niels-Oke Schwien ${ }^{2}$ and Markus Töpfer ${ }^{3}$ \\ German Aerospace Center (DLR e.V.), Cologne, Germany
}

\begin{abstract}
Voice Communication Systems for Mission Control Room environments are essential for multiple group communications in parallel. Current systems have a high demand on the user's multitasking abilities. To support users, these systems have recording and playback capabilities but are not optimized for usability and not integrated in the user's workflow. Playback capabilities are undervalued within current system designs. Within this paper we will focus on playback functionalities and propose a solution with a simplification of the playback workflow using a gesture controlled and time-domain based visualization for recordings. In this way users are able to replay missed or important communication parts of a specific user, for example an astronaut without the need of repetitive transmission. Combining real time functionality and providing access to past information via recordings within the same user interface may resolve conflicts between listening to live and previous communications in parallel. Interacting with recordings using a time domain based playback interface may enhance operation support, for example during debriefings, for staff training or as a source for verifying and validating unclear information. The shown solution may be used as an input for next generation system designs, which may improve capabilities and extend scenarios of playback for operation support.
\end{abstract}

\section{Introduction}

$\mathrm{V}^{\mathrm{s}}$ oice Communication Systems for Mission Control Room environments are reliable, resilient and failureresistant. They work in 24/7 use case scenarios and provide capabilities for group communication (conferencing). Their is the provision of multiple group communications in parallel. The term Voice Loop is used to describe everything related to the transmission of the communication and is a synonym for a telecommunication conference. System users have to monitor many Voice Loops in parallel. Monitoring more than one conversation at the same time requires multitasking abilities and is challenging even for experienced control room crew members. In case of voice traffic on several Voice Loops at the same time, communication parts may be not clearly understood. Playback of unclear communication parts may be used to ensure the communication is understood without the need for repetitive transmission or questions regarding the repetition of the previous communication.

Playback in Voice Communication Systems (VoCS) for Mission Control Rooms is used for two distinct purposes, short-term playback to replay misunderstood parts, for repetitive output of a current communication as well as for long-term playback of stored communication.

Current VoCS provide playback features for both purposes, but integration within the user's terminal is done as an add on functionality and the implementation itself is not optimized to separate the streams of the Voice Loops.

Within this paper, we will describe an improvement to this specific functionality in form of a gesture controlled time domain based playback user interface. Adding a time domain to the default user interface for Voice Loop selection places playback access directly in the natural system view. Access to recordings is as simple as scrolling

\footnotetext{
${ }^{1}$ Student, Simulation and Software Technology | Distributed and Component Software, Rosa-Luxemburg-Strasse 2 10178 Berlin.

${ }^{2}$ Student, Simulation and Software Technology | Distributed and Component Software, Rosa-Luxemburg-Strasse 2 10178 Berlin.

${ }^{3}$ Scientific Researcher, Space Operations and Astronaut Training | Communication \& Ground Stations, Münchener Straße 20, 82234 Weßling.
} 
the current interface back in time. The integration of such a concept may enhance the efficiency of ongoing communication. Functionality and concept of the gesture controlled time-domain playback functionality and system integration will be described in detail in section II.

Section III describes a multi touch Android prototype, which was used to evaluate the idea and concept in a user test. In section IV we show the concept evaluation performed at the German Space Operation Center in Oberpfaffenhofen, using two different user groups. The first test group consists of longtime VoCS users and focus for this group is the potential improvement for operations. The second test group consists of students. That group is used as a reference group of digital natives, which may become VoCS operators within Mission Control in the next years. Here we emphasized on evaluating usability and expectations in terms of user experience from next generation operations personal. Results of the tests are shown in section $\mathrm{V}$, which is followed by a concluding discussion of the presented solution. Before we start describing the concept of time-domain based user interfaces we will shortly introduce a bit further into the technical area with a state of the art and a shortcomings overview.

\section{A. State of the Art}

The state of the art description is based on the VoCS of the German Space Operations Center (GSOC) used to support the Columbus Mission of the International Space Station. This system is one of the latest installations in Europe and was introduced in 2012.

The user terminal for the selection of Voice Loops is a resistive industrial touch screen. These screens are not as responsive as modern capacitive touch screens in consumer electronic. A terminal screen shows a grid of Voice Loops, these are accessible with the selected role during login. Access to a Voice Loop is restricted by a role based access control (RBAC). Pressing on a Voice Loop button toggles the state from non-participating to monitoring (listening). In addition to the Voice Loop grid, another area with buttons for system actions is provided. One of these buttons is for short-term playback. Pressing this button will open an overlay window showing all available shortterm recordings. Short-term recording is implemented as file based recording of the sum signal of all currently active communications on the device. These files contain the recorded audio sent to the user's headphones in intervals of activity and contain up to 60 minutes audio data of past communications. Within the playback interface, the recorded files are named with the timestamp of creation, as well as with a list of Voice Loop names with audio activity during the recording process.

Long-term playback is not accessible from terminals. For long-term playback a dedicated archive application is used. Access to this application is not granted to users of the voice system. To get a recording from the archive, a user needs to make a request containing information about time span and Voice Loops using an external tool. These requests are processed by a system engineer with administration privileges, who exports the requested recordings manually and hand them over to the requesting user on a portable storage media. Because of internal regulations exported recordings can only be handed over to the users, if the export system authority verified the export. For the Columbus Mission this authority is the ESA Mission Director. Control about the data is lost for each long-term playback request.

The whole process for a long-term playback is as follows:

1. User requests the long term records using the request tool

2. Ground Control receives the request and coordinates with the ESA Mission Director in charge

3. If the request is granted, Ground Control coordinates an export of the data with the system engineers

4. System engineers export the data and hand it over to Ground Control

5. Ground Control hands over the recordings to the user who requested them

This process involves three different entities which process the request, as the request itself will break data security in terms of:

1. Data confidentiality (no more control who gets which data)

2. Data authenticity (no more control if the audio is really the requested audio)

3. Data accountability (no longer ensured who distributes the data)

\section{B. Shortcomings}

Short-term recording is a sum signal of all active audio and not Voice Loop based. The interface and naming scheme is not intuitive and requires paying attention to play back a missed part of communication. The user needs to identify short-term recordings based on file names, which are named differently than the communication part of interest (Voice Loop is part of a list). Long-term recording is not accessible from a user terminal. The current longterm solution is a data security issue. The mitigation workflow for long-term playback requires a long time span between request of data and access to data. 
Over the last years, tablets, smartphones and similar devices made multi-touch based controlled applications omnipresent. Mass distribution of contemporary touch devices leads to conditioning of users, who expect to interact with capacitive multi touch based interfaces. Potentially, soon-to-be mission control room crew members, who grew up as digital-natives using devices with modern touchscreens, will miss the learned natural interaction design patterns like pinching, swiping or scrolling.

\section{Time Domain based User Interface Concept}

The user interface of VoCS is used to select Voice Loops for future communications. Extending it with a time domain based interface to replay past communication could probably increase usability, user satisfaction and solve issues with data security.

The concept rests upon modern interaction methods of mobile platforms such as Android. This concept is our approach to evaluate how playback functionality could improve usability of the whole system and optimize user's workflow. It introduces easily understandable timelines for Voice Loop based recordings. Timelines may be a good extension point for time based features and offer space for visual annotations and recordings metadata.

\section{A. Design Considerations}

The concept shall be an all in one short- and long-term playback interface. The main objective is to design the playback context as easy to use as possible. In stressful situations users have to change from the live communication context to the playback context with no effort. Interaction within the application shall be based on multi-touch gestures. The interaction space (Fig. 1) shall cover the whole playback context to make the gestures usable in every situation. The displayed metadata shall simplify the process of searching for time spans of interest.

Searches shall be possible based on Voice Loops, roles, users and time spans. Long-term playback capabilities shall make use of annotations and time based features like bookmarks.

Core design questions for a long-term playback user interface functionality are:

1. How to navigate a timeline in a smooth, intuitive and fast way?

2. How to show related information sets for a time frame?

3. Which information sets are most important depending on the time scale?

The user interface needs to be as simple as possible. To reduce the complexity, the interface contains two areas, one area to control the playback functions and another area to select voice loops of interest.

\section{B. Core Functionality}

The user interface consists of timelines, each is representing a Voice Loop. Navigating in time means to manipulate the time frame of the timelines. With such an approach it is possible to scroll linearly back in time with horizontal scroll gestures. Stored recordings will be shown within the timeline. Swiping from left to right will scroll back in time and show available recordings in the viewport. Another way is to use input text fields to jump to a different point in time based on the given input. Both solutions are commonly used for time based navigation.

Long-term playback whereas needs another approach. In this concept long-term navigation means to manipulate

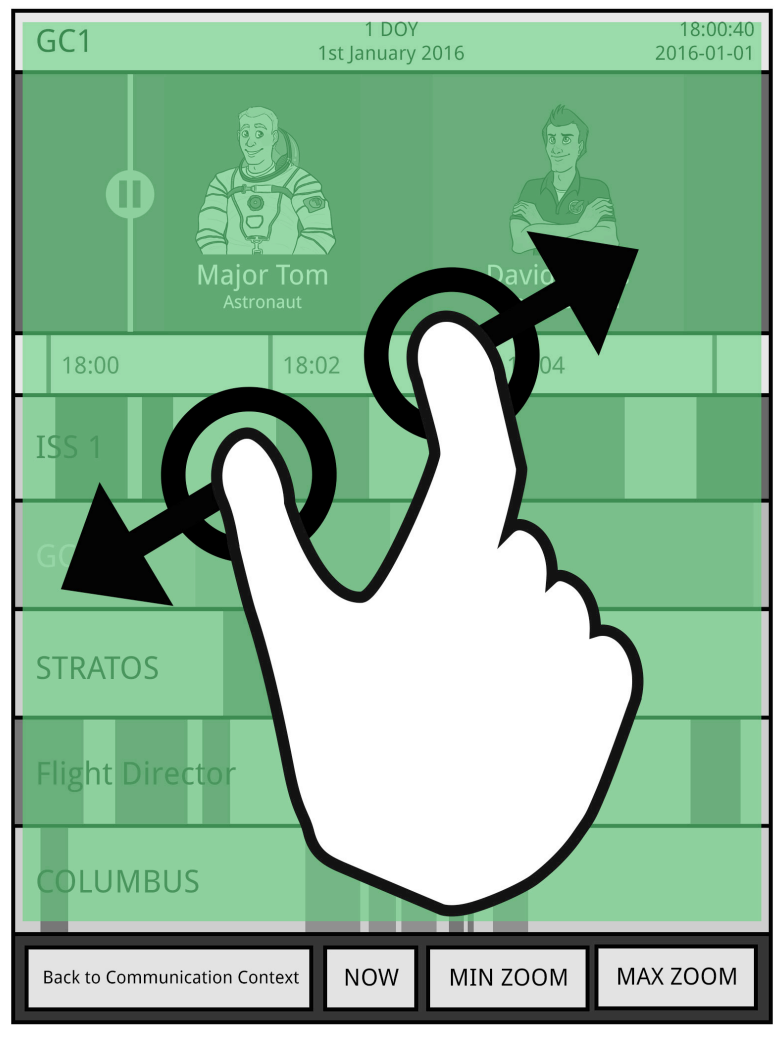

Figure 1. Interaction space. timeline's time scale by zooming in and out the time frame.

Pinching the timeline can be done to zoom the time frame. Most users may remember this as zoom gesture for pictures on multi-touch devices. 


\section{Dynamic Timeline Annotation}

Swiping back over hours, days, weeks or even longer time spans with a linear time resolution is not usable. For record access of long-term recordings time inputs may be used to set the viewport of the application to a different point in time. With Dynamic Timeline Annotation navigating through time is simple. Dynamic Timeline Annotation divides the time frame of the timeline into time units. Time units of interest for a playback interface are minutes, hours, days, weeks, months and years. When a user zooms the viewport out, the time frame shown gets wider until a specific point and subsequently jumps to the superior time unit. Therefore the zooming process is separated into two parts. The first part is a traditional zoom, which increases the time frame shown within the viewport. The second part of the process is the jump to the superior time unit. Zooming out increases the resolution level and zooming in decreases the level.

As soon as the viewport shows more than one hour, individual records will no longer be displayed and the system is switching to a label based view. A user can single tap a date on the timeline with a represented time frame higher than 10 minutes to jump directly into the tapped, inferior time unit (Fig. 2). To jump to the highest (long term) and lowest time unit (short term) additional actions are provided in the menu bar. One hour as scale for the switch to the label based view may be seen as the switch from short term to long term context navigation.

With Dynamic Timeline Annotation no keyboard entries or workflow breaking dialogs distract the user. This navigation method merges short- and long term playback. It does not matter anymore if a communication search is for the recent or further past.

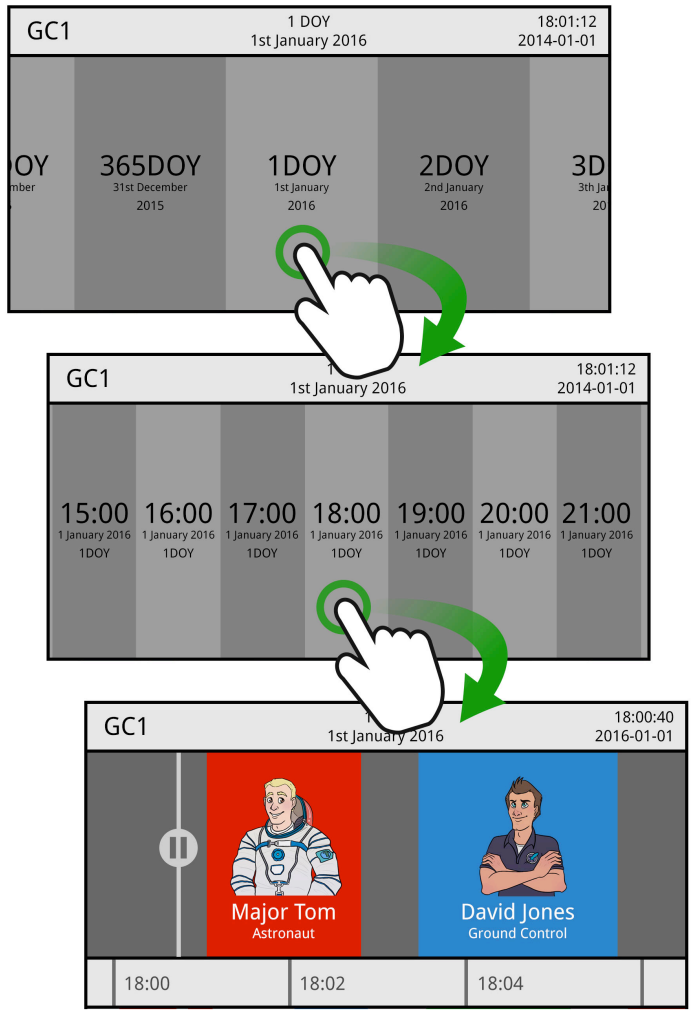

Figure 2. Dynamic Timeline Annotation behaviour.

\section{Bookmarks}

Bookmarks can be used to tag a specific point in time or recording with a label. Bookmarking a point in time means storing a point in time with additional information. That may be the content of the communication e.g. a bookmark labeled with "Repair of water pump" or used to tag a specific activity in a mission's context. That opens up new use cases for debriefings or shift handovers. Bookmarks may be private or public. Private bookmarks can only be accessed by the user who stored it. Public bookmarks are related to a Voice Loop and shared with all users in the system. Using bookmarks may simplify information exchange related to recoding data sets.

Sharing information about recordings of interest is currently done by handing over the time of the recording. The information receiver needs to perform a search for the recording within the playback interface before they is able to access the audio data. A shared labeled bookmark would eliminate that search, as it is a link to the point in time with the recording of interest.

\section{Prototype Description}

Android was used for a prototype implementation. Android is the biggest consumer class platform for smartphones and tablets. The Android runtime has great capabilities for touch interactions and is fast to prototype with. 
When the playback context is opened, the voice communication is displayed in timelines (Fig. 3). Timelines divide the screen into active and inactive timeline types. On start-up, the time frame of the timelines shows the last two minutes. This is a useful default time span for the short-term playback use case. All timelines show the same time frame. The time frame can be adjusted by gestures on all timelines. Colored rectangles visualize recordings. The color information is indicating the speaker's role (e.g. astronauts red and Flight Directors blue). A label with the name of the Voice Loop is displayed on the left of a timeline.

The active timeline is used to provide additional information about time frame and recordings. It represents the interface for interaction with the playback controls. The active timeline consists of a header, recording information area and footer.

In addition to the general information the header shows a date indication (DOY and calendar date) in the center and the playback marker's current date and time on the right. Within the footer time annotation is shown.

The recordings in the active timeline show additional role data like name and avatar.

Within the active timeline a marker is moving to visualize the current playback time. To move the marker a user can single tap or drag it to another point in time. The marker behaves like a media player and has a play/pause button. The marker moves in real time through the timeline. Once the marker intersects a recording, this recording gets played.

To change the active timeline a user can single tap the intended inactive Voice Loop's timeline. As soon as they tapped the timeline it will be displayed in the active timeline and the inactive timeline manifestation will be greyed out.

With swiping and pinching the displayed time frame can be manipulated depending on the gesture's intensity.

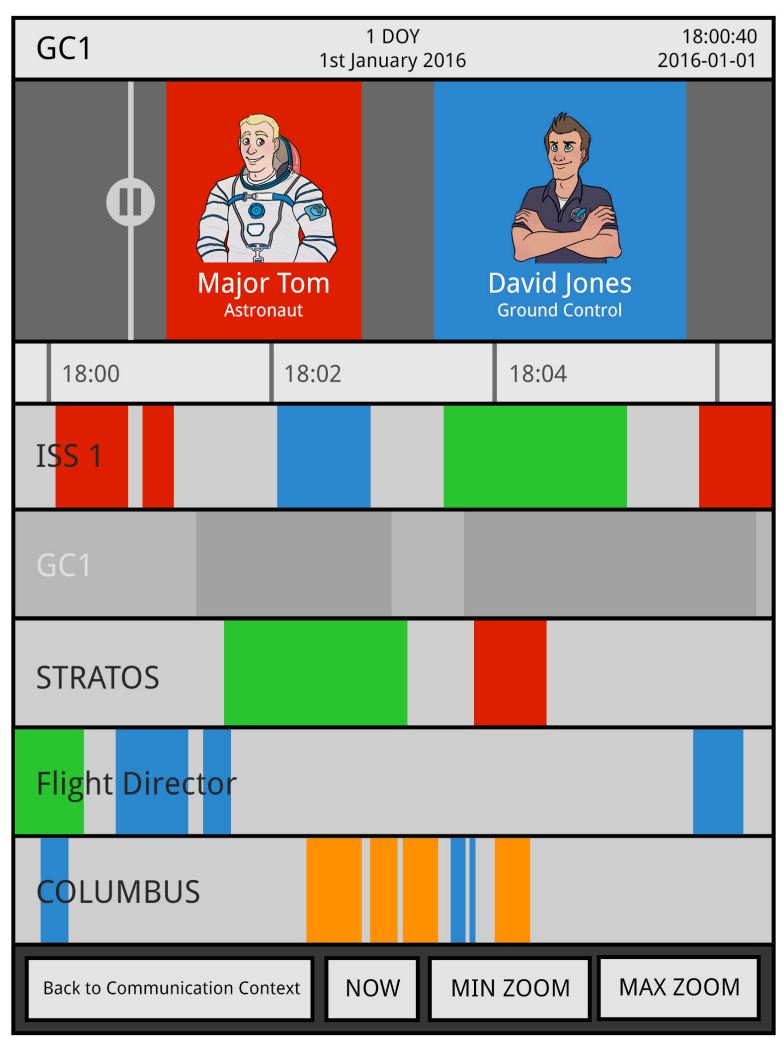

Figure 3. User Interface Prototype.

Swiping from left to right moves the time frame to the left, swiping from right to left moves it to the right. Pinching from the outside to the inside extends the displayed time frame, pinching from the inside to the outside shrinks it. The menu bar with all buttons for actions is on the bottom of the screen. That way the hand of the user is not covering the rest of the screen during button presses.

To playback all selected Voice Loops using a sum signal is an easy extension of the idea, but out of scope for the current analysis.

The prototype shall be used to verify potential enhancements of operations using dedicated Voice Loop based playbacks and considering modern user experience design patterns.

\section{User Test}

A user test session was executed at the German Space Operations Center (GSOC) in Oberpfaffenhofen, Germany. The prototype was tested to gather information about potential increases in usability, user experience and overall improvement of the playback functionality. The test was executed with two different test groups: one group of students who are digital natives and one group of longtime users who operate VoCS on a daily basis.

As general test method "Thinking-Aloud" was used. That means, while executing the test, each participant was encouraged to openly communicate thoughts about the steps they were currently performing. After the test we evaluated the user experience with an interview.

The idea behind the user tests was to verify the following questions:

1. Is the time domain based visualisation of recordings an understandable concept for possible soon-to-be and longtime VoCS users?

2. Are interactions on the time domain based visualisation intuitive to use? 
3. Are interactions on the time domain based visualisation more efficient than old patterns like inputs via dialogs?

4. Can Voice Loop based playback improve the user experience for longtime VoCS users?

Our evaluation is based on the ability of users to find their own way through requested tasks. We don't aim for a solution where every problem is solvable by each user in the first guess, we aim for a solution where the second guess about the correct solution is good enough.

\section{A. Description Test Group 1: Students}

The test scenario consisted of instructed tasks where students had to use the prototype to start playbacks of different Voice Loops at different points in time. Some points in time were chosen from the nearer past, some from the further past. We tested the students with the "Thinking-Aloud"-method for the questions if time domain based visualization is an understandable concept, and if the visualization is intuitive to use.

To evaluate if touch based gestures are more efficient than inputs via dialogs, we divided the group of students into two reference groups. Group A had to navigate with a dialog date picker. Group B had to navigate with multi touch gestures.

Each participant was performing the following test sequence:

1. Intro briefing about the prototype

2. Time to look around in the user interface

3. Stop "Thinking-Aloud"-method and start time tracked testing

4. Instruction to navigate to the first date and to playback a short term record

5. Instruction to replay the record of the first date again

6. Instruction to navigate to a second date and to playback a long term record

7. Instruction to navigate to a third date and to playback a short term record

8. Instruction to navigate to a fourth date and to playback a long term record

9. Interview

We took the time between each navigation step of each participant.

\section{B. Description Test Group 2: Longtime VoCS Users}

The test scenario for the longtime VoCS users consisted of instructed tasks where they had to use the prototype to start playback of different Voice Loops. Focus of this test set was to evaluate if a Voice Loop based playback functionality could improve the user experience in comparison to the currently deployed system at GSOC. A concluding interview was performed to survey the user about their personal experience with the prototype. Main focus of this test set was observing the interaction with the prototype.

Each participant was performing the following sequence:

1. Intro briefing about the prototype

2. Time to look around in the user interface

3. Instruction to navigate to the first date and to playback a short-term record

4. Instruction to replay the record of the first date again

5. Instruction to navigate to a second date and to playback a long-term record

6. Instruction to navigate to a third date and to playback a short-term record

7. Instruction to navigate to a fourth date and to playback a long-term record

8. Interview

Interview questions used are:

1. Is the prototype intuitive to use?

2. Is the prototype fun to use?

3. Do you recognize some interaction patterns of other applications?

4. Is the timeline concept practical?

5. What is the difference between the big timeline and the small timelines?

6. Is a Voice Loop based playback based on timelines a good concept?

7. Can you imagine a situation where the prototype would improve your workflow?

8. Have you ever been depended on the VoCS playback function?

9. Have you ever needed a long-term recording, but you didn't had access to it? 


\section{Test execution}

During the task we video recorded the participants and especially the participant's hands in combination with the device. The prototype was tested as an independent application without live traffic. Each participant was tested separately. Participants were briefed with information about the prototype and the test procedure. After the introduction they had to perform the test sequences and to navigate to different points in time.

\section{Results}

The first and most foremost outcome of the tests is a very positive user feedback, over the whole test group. Each participant was able to use the time domain based approach we introduced.

All tested students and longtime users were interested in the interface and familiar with touch interaction on capacitive touch screens. Most longtime users pointed out that they are not satisfied with the current interaction on the resistive industrial touch screens. Even older crew members are habituated to touch controls in private smartphones. It was possible for all users to select inactive timelines and play back recordings at a specific point in time without help. Almost all participants recognized the colored rectangles on the timelines as recordings of different roles. The first thoughts of some participants were the colors depend on different users, but they soon realized the role reference.

Navigation on the timelines was fast for both short- and long-term playback. Scrolling back in time was intuitive and found out by each participant immediately. Whereas a hint from the instructor was needed for zooming the time frame via the pinch gesture. With the knowledge of scroll and pinch gestures the participants navigated confidently to different points in time. Students of group A (without the pinch gestures) were less satisfied when they had to navigate to past communications. Students of group B reached the points in time listed in the test sequence faster and pointed out they enjoyed the usage of the prototype more often than group A.

Longtime VoCS users are heavily conditioned on the current installed VoCS and most widely rejected the playback functionality because of usability issues described within the state of the art section. Longtime VoCS users feedback had in common mentioning the debriefing use case idea. Interestingly everyone tested, told us about it. They liked the interaction via gestures. They said that the loop based playback is exactly what they need in most of the playback cases.

\section{Discussion}

Time domain based interfaces were recognized as very intuitive and as a natural way to use playback capabilities independent of the depth of previous knowledge about VoCS in mission control. Students as well as longtime VoCS users gave a very positive feedback about the concept.

In the current system the playback functionality is not as powerful as it could be. To support users in their work the concept of a time domain based visualization is understandable. Usability engineering seems to be underestimated in voice system applications. Transferring ideas from interaction design patterns of consumer products helps to create a better user experience. Users independent of age are conditioned to interaction concepts of consumer electronics. The user test shows that new interaction methods are needed in control room environments. The users are tired of resistive industrial touch screen interfaces and want the same gestures in the control room as on their smartphones. Based on the test results the navigation through gestures seems to be more efficient than a navigation using a date picker dialog.

We expected that for long-term users it must be self-evident to hear live and past communication in parallel, but indeed the opinions about mixing live and past conversations were diverging. It depends on the mission the user is working on, if parallel communication is an advantage or not. All interviewed test participants came up with ideas and use cases for short- and long-term playback by themselves and liked the idea of listening to recordings per loop. User experience in VoCS is indeed improvable by a Voice Loop based playback.

An all-in-one short- and long-term application reduces overhead and operational costs, because long-term playback can be directly accessed on terminals without the need to involve system engineers and system authorities. Recordings do no longer get exported and data confidentiality, data authenticity and data accountability can be granted.

\section{Conclusion}

Current VoCS users are tremendously interested in a usable interface for playback. The tested time domain based interface is one solution to make the short-term playback a usable extension for VoCS. Better integrated Voice Loop based playback functions increase the user's efficiency by avoiding to ask for repetition on a Voice Loop and could lead to an improved radio discipline. Long-term capabilities depending on roles and missions are desirable for 
debriefing, mission evaluation and could create additional new use cases. New voice systems in mission control room environments have to be based on multi gesture controls to satisfy user experience nowadays.

User experience testing should be done for new system designs within a mission control room context. Prototyping and user feedback testing is highly appreciated by the audience group.

\section{References}

Books

${ }^{1}$ Krug , S., Don't Make Me Think: A Common Sense Approach to Web Usability, $3^{\text {rd }}$ ed., New Riders Verlag, Berkeley, 2013, Chap. 9.

${ }^{2}$ Wigdor, D. and Wixon, D, Brave NUI World: Designing Natural User Interfaces for Touch and Gesture, ELSEVIER B.V., Amsterdam, 2011, Chap. 1, 2, 4, 21, 22. 